Enferm Bras 2020;19(6):492-501

https://doi.org/10.33233/eb.v19i6.4429

\title{
ARTIGO ORIGINAL \\ Uma análise retrospectiva dos erros de medicação ocorridos em unidades de transplante de medula óssea
}

Simone Carreiro Brasil, M.Sc. ${ }^{*}$, Simone Pereira Lermontov, M.Sc. ${ }^{*}$, Renato Azevedo dos Santos Filho* $^{\star \star *}$, Ana Cristina da Silva Rangel ${ }^{\star \star \star *}$

${ }^{\star}$ Enfermeira da Comissão de Controle de Infecção Hospitalar, ${ }^{* *}$ Enfermeira, Coordenadora de Pesquisa Clínica do Centro de Transplante de Medula Óssea - Instituto Nacional de Câncer José Alencar Gomes da Silva, ${ }^{* * *}$ Enfermeiro do Centro de Transplante de Medula Óssea do INCA, ****Supervisora de Enfermagem do Centro de Transplante de Medula Óssea -Instituto Nacional de Câncer José Alencar Gomes da Silva

Recebido em 28 de outubro de 2020; aceito em 20 de dezembro de 2020.

Correspondência: Simone Pereira Lermontov, Instituto Nacional de Câncer José Alencar Gomes da Silva, Praça da Cruz Vermelha, 23 Centro 20230-130 Rio de Janeiro RJ

Simone Carreiro Brasil: sbrasil@inca.gov.br

Simone Pereira Lermontov: simone.lermontov@inca.gov.br

Renato Azevedo dos Santos Filho: renavedo@gmail.com

Ana Cristina da Silva Rangel: arangel@inca.gov.br

\section{Resumo}

A terapia medicamentosa no transplante de medula óssea (TMO) é complexa, os pacientes são expostos a protocolos de tratamentos prolongados, com inúmeros medicamentos, o que aumenta o risco do erro de medicação (EM). Objetivos: Identificar os erros de medicação, os profissionais envolvidos, os medicamentos envolvidos, as causas da ocorrência, as consequências para o paciente e as ações de melhoria implementadas. Métodos: Trata-se de um estudo observacional retrospectivo com abordagem quantitativa, o período de coleta dos dados foi de janeiro de 2008 a janeiro de 2018. Resultados: Foram identificados 42 erros de medicação. Os agentes antineoplásicos, imunossupressores e antibióticos foram os mais frequentes no EM. O fator humano foi a causa da ocorrência do EM mais frequente (69\%). Nas consequências para os pacientes, foram identificados eventos adversos: leves $82 \%$; moderados $14 \%$; graves $2 \%$ e fatais $2 \%$. As ações de melhoria implementadas para prevenção foram: treinamento da equipe; elaboração de protocolos padrões; orientação de pacientes e acompanhantes; revisão do sistema eletrônico de prescrição; dupla checagem da prescrição; aprazamento por enfermeiras com tempo de experiência; entre outras. Conclusão: Os EM põem em risco a segurança do paciente, as causas dos EM foram multifatoriais e as consequências em alguns casos foram graves ou fatais.

Palavras-chave: transplante de medula óssea, erros de medicação, segurança do paciente.

\section{Abstract \\ A retrospective analysis of medication errors occurred in bone marrow transplantation units}

Drug therapy in bone marrow transplantation (BMT) is complex, the patients are exposed to protocols of prolonged treatment, with numerous medications, which increases the risk of medication error (ME). Objectives: To identify medication errors, the professionals involved, the drugs involved, the causes of the occurrence, the consequences for the patient, and the improvement actions implemented in a BMT unit. Methods: This is an observational retrospective study with a quantitative approach. The data collection period was from 2008 to 2018. Results: We identified $42 \mathrm{ME}$. Antineoplastic agents, immunosuppressants, and antibiotics were the most frequent in ME. The human factor was the most frequent cause of ME $(69 \%)$. The consequences for patients identified were adverse events, being mild $82 \%$; moderate $14 \%$; severe $2 \%$ and fatal $2 \%$. The improvement actions implemented for prevention were: training the team; elaboration of standard protocols; guidance for patients and companions; review of the electronic prescription 
system; double prescription check, among others. Conclusion: The ME that endanger patient safety were identified, in which the antineoplastic agents were the drugs most likely to damage.

Keywords: bone marrow transplantation, medication errors, patient safety.

\section{Resumen \\ Un análisis retrospectivo de los errores de medicación ocurridos en unidades de trasplante de médula ósea}

La farmacoterapia en el trasplante de médula ósea (TMO) es compleja, los pacientes están expuestos a protocolos de tratamiento prolongado, con numerosos medicamentos, lo que aumenta el riesgo de error de medicación (EM). Objetivos: Identificar los errores de medicación, los profesionales implicados, los fármacos implicados, las causas de la ocurrencia, las consecuencias para el paciente y las acciones de mejora implementadas. Métodos: Se trata de un estudio observación retrospectivo con enfoque cuantitativo, el período de recolección de datos fue de enero de 2008 a enero de 2018. Resultados: Se identificaron 42 errores de medicación. Los agentes antineoplásicos, inmunosupresores y antibióticos fueron los más frecuentes en la EM. El factor humano fue la causa más frecuente de EM (69\%). Las consecuencias para los pacientes, se identificaron eventos adversos: leve $82 \%$; moderado $14 \%$; severo $2 \%$ y fatal $2 \%$. Las acciones de mejora implementadas para la prevención fueron: capacitación del equipo; elaboración de protocolos estándar; orientación para pacientes y acompañantes; revisión del sistema de prescripción electrónica; control de prescripción doble; aprobación por enfermeras con tiempo de experiencia; entre otras. Conclusión: La EM puso en riesgo la seguridad del paciente, las causas de la EM fueron multifactoriales y las consecuencias en algunos casos fueron graves o fatales.

Palabras-clave: trasplante de médula ósea, errores de medicación, paciente seguridad.

Introdução

Nos últimos anos, inúmeros foram os esforços e mobilizações por parte das instituições e dos profissionais de saúde, visando estratégias para prevenção e redução dos danos aos pacientes[1]. A segurança do paciente é uma demanda que afeta os países em todos os níveis de desenvolvimento, contudo os dados relacionados a essa questão ainda são subestimados, principalmente, nos países em desenvolvimento [2].

A fim de organizar os conceitos e definições sobre segurança do paciente, foram propostas medidas para reduzir os riscos e minimizar os danos e eventos adversos decorrentes. Em 2004, a Organização Mundial da Saúde (OMS) criou a Aliança Mundial para Segurança do Paciente, que passou a chamar-se Patient Safety Program (Programa de Segurança do Paciente) $[2,3]$.

A Aliança Mundial para Segurança do Paciente mobiliza iniciativas, difunde conhecimentos e propõe soluções relacionadas ao tema [3,4]. Uma das ações centrais do programa é denominada Desafio Global, uma estratégia que define foco na minimização de um determinado risco de dano ao paciente [3,4]. Assim, em 2017 reconhecendo a grande ocorrência de incidentes e eventos adversos relacionados ao uso de medicamentos, tanto em países desenvolvidos como em desenvolvimento, o Programa de Segurança do Paciente da OMS definiu o Terceiro Desafio Global que tem como objetivo abordar as fragilidades nos sistemas de saúde que levam a erros de medicação (EM) e os graves danos que podem causar [2,3].

O Brasil, em consonância com a mobilização mundial, instituiu em 2013, o Programa Nacional de Segurança do Paciente, estabelecendo um conjunto de protocolos básicos, buscando a prevenção e redução de incidentes relacionados ao cuidado em saúde. Cabendo destacar, as recomendações quanto a segurança na prescrição, dispensação e administração de medicamentos, haja vista, entre outras, a magnitude dos erros e eventos adversos decorrentes da falta de ações neste sentido no país $[4,5]$.

No âmbito do transplante de medula óssea (TMO), a terapia medicamentosa é complexa, os pacientes são expostos a protocolos de tratamentos prolongados, com inúmeros medicamentos. O TMO é dividido em fases: pré-transplante, transplante (condicionamento e infusão) e pós-transplante. Dessas fases destaca-se o condicionamento, que consiste na administração de altas doses de quimioterápicos. Durante o mesmo inicia-se a terapia adjuvante com antieméticos, anti-histamínicos, corticosteróides, analgésicos, imunossupressores, protetores gástricos e antimicrobianos específicos com o propósito de evitar, reduzir ou aliviar reações adversas decorrentes da quimioterapia ou prevenir complicações, tais como, mucosite; 
doença do enxerto contra o hospedeiro (DECH); doença veno-oclusiva hepática (DVOH); cistite hemorrágica; entre outras [6].

Os pacientes submetidos ao transplante de medula óssea representam um grupo de alto risco para o EM, visto a quantidade de medicamentos que estão expostos durante o tratamento e principalmente durante o condicionamento. Logo, o conhecimento dos enfermeiros sobre os efeitos terapêuticos e as reações adversas podem prevenir o EM, minimizar os danos e promover a segurança do paciente [6].

Sendo assim, este estudo tem como objetivo: identificar os erros de medicação, os profissionais envolvidos, os medicamentos envolvidos, as causas da ocorrência, as consequências para o paciente e as ações de melhoria implementadas em uma unidade de Transplante de Medula Óssea.

\section{Material e métodos}

Trata-se de um estudo observacional retrospectivo com abordagem quantitativa, realizado em uma unidade de Transplante de Medula Óssea. Esta unidade faz parte da Rede de Hospitais Sentinela (RHS) da Anvisa. O programa foi criado em 2001, em parceria com uma rede nacional de hospitais públicos e privados, para notificação de não conformidades no uso de produtos para a saúde, o que inclui os medicamentos. Em 2008, com processo de acreditação hospitalar, a notificação de riscos assistenciais relacionados às falhas nos processos de trabalho foi implementada em nossa unidade, ampliando o escopo da gestão de risco. Desde então, a ocorrência de uma não conformidade, desvio de qualidade de produtos para a saúde e suas consequências, assim como, os eventos relacionados às falhas nos processos assistenciais são comunicados ao gerente de risco.

Esta comunicação é realizada por meio de formulário padronizado que possibilita o início das ações corretivas ou preventivas. A comunicação de evento é realizada, pelos membros da equipe, ao gerente de risco por meio do formulário impresso, discriminando o tipo e o contexto da ocorrência. Segue-se uma verificação, por meio da leitura de registros do prontuário e coleta de dados, além de abordagem aos profissionais envolvidos, complementando a busca de informações. Após a conclusão da análise do caso é dado o direcionamento cabível, visando o planejamento das ações de melhoria, bem como implementação das mesmas.

Os EM foram identificados das fichas de notificação de incidentes e eventos do serviço, que fazem parte do banco de dados da gerência de risco. O período de coleta dos dados foi de janeiro de 2008 a janeiro de 2018. As variáveis de interesse foram: faixa etária; local onde ocorreu o EM; tipo de erro; via de administração; categoria profissional envolvida; causas da ocorrência; medicamento envolvido; consequências para o paciente e ações de melhoria.

A classificação dos tipos de EM foi realizada pelos autores, utilizando a taxonomia espanhola, elaborada pelo grupo de trabalho Ruiz-Jarabo 2000 [7]. A classificação espanhola pode contribuir para a organização e aprimoramento das informações colhidas por instituições de saúde brasileira que já trabalham com foco na segurança dos pacientes e prevenção de erros.

Os dados foram compilados em planilha do programa Excel $\AA^{\circledR}$, preparada especificamente para o estudo e foram analisados por meio de estatística descritiva, com distribuição de frequências absoluta e relativa. O estudo seguiu os preceitos éticos da Resolução 466/12[8] e foi aprovado pelo Comitê de Ética em Pesquisa: CAAE - 87760318.8.0000.5274.

\section{Resultados}

Foram analisadas 87 fichas de notificação de incidentes e eventos na unidade de TMO, sendo identificados 53 eventos com medicamentos (61\%). Destes, 11 foram classificados como quase erros e 42 como erros de medicação. Em relação à faixa etária $46 \%$ dos EM ocorreram em crianças (3-11 anos) e adolescentes (14-17 anos), 54\% em adultos, sendo $43 \%$ dos casos em homens. Não houve registro da idade e do gênero do paciente em $17 \%$ dos casos. Quanto ao local da ocorrência do EM, 90\% foram na unidade de internação, $7 \%$ no hospital dia e 3\% no domicílio dos pacientes.

Os profissionais envolvidos no EM, foram enfermeiros (38\%), farmacêuticos (36\%), médicos (17\%), residentes de enfermagem (2\%) e cuidadores/familiares $(7 \%)$. Quanto à via de administração, 59\% dos EM ocorreram pela via endovenosa. Os medicamentos envolvidos estão descritos na tabela I, sendo os agentes antineoplásicos, imunossupressores e antibióticos os mais recorrentes no EM. 
Tabela I - Categoria Farmacológica - Medicamentos envolvidos no erro de medicação, Rio de Janeiro, 2018.

\begin{tabular}{lll}
\hline Classe fammacológica & Número de casos & Exemplos de medicamentos \\
\hline Antibióticos & 3 & Cefepime (1); Polimixina B (1) \\
\hline Agentes antineoplásicos & 14 & $\begin{array}{l}\text { Bussúan (3); Cidofos f́mida (3); } \\
\text { Etoposido (1); M etotrexato (1) }\end{array}$ \\
\hline Agentes imunossupressores & 8 & $\begin{array}{l}\text { Micofenolato (1); Globulinatimocitica } \\
(1) ; \text { Cidosporina (4); Imunoglobulina (2) }\end{array}$ \\
\hline Agentes anticoagulantes & 1 & Enoxoporina sódica (1) \\
\hline Agentes antimicrobianos & 2 & Fluconazol (1); M etronidazol (1) \\
\hline Agentes opioides & 2 & Fentanil (1); Codeina (1) \\
\hline Antieméticos & 2 & Metodopramida (1);Ondansetrona (1) \\
\hline Diuréticos & 1 & Vasopressina (1) \\
\hline $\begin{array}{l}\text { Fator estimulante de } \\
\text { colônias }\end{array}$ & 2 & Filgrastim (2) \\
\hline Eletrólitos & 2 & Cloreto de potássio (2) \\
\hline Vitaminas & 2 & Fololinato de Cálcio (2) \\
\hline Protetor Urológico & 1 & Mesna (1) \\
\hline S/D & 2 & Não descritos \\
\hline Total & 42 & Medicamentos envolvidos em EM \\
\hline Fonte: Dados da Gerência de Risco do Centro de Transplante de Medula Óssea, 2008-2018.
\end{tabular}

Na tabela II estão descritos os tipos de erros de medicação, sendo os mais frequentes: a omissão de dose ou do medicamento (26\%); dose errada (24\%) e erro de preparo/manipulação e/ou acondicionamento (10\%).

Tabela II - Tipos de erro de medicação, Rio de Janeiro, 2018.

\begin{tabular}{|c|c|}
\hline \multicolumn{2}{|l|}{ Tipos de erros de medicação } \\
\hline Medicamento errado & $\mathrm{N}(\%)$ \\
\hline - Prescrição inadequada do medicamento & $2(5)$ \\
\hline $\begin{array}{l}\text { - Medicamento não indicado/ não apropriado } \\
\text { para o diagnóstico que se pretende tratar }\end{array}$ & 1 (2) \\
\hline - Medicamento desnecessário & $1(2)$ \\
\hline \multicolumn{2}{|l|}{ Omissão de dose ou do medicamento } \\
\hline $\begin{array}{l}\text { - Falta de prescrição de um medicamento } \\
\text { necessário }\end{array}$ & $4(10)$ \\
\hline - Omissão na dispensação & $2(5)$ \\
\hline - Omissão na administração & $5(12)$ \\
\hline \multicolumn{2}{|l|}{ Dose enrada } \\
\hline - Dose maior & $4(10)$ \\
\hline - Dose menor & $5(12)$ \\
\hline - Dose extra & $1(2)$ \\
\hline Frequência de administração errada & $1(2)$ \\
\hline $\begin{array}{l}\text { Erro de preparo, manipulação e/ou } \\
\text { acond icionamento }\end{array}$ & $4(10)$ \\
\hline Técnica de administração errada & 1(2) \\
\hline Via de administração errada & $1(2)$ \\
\hline Velocidade de administração errada & $1(2)$ \\
\hline Horário enrado de administração & $2(5)$ \\
\hline Paciente errado & $2(5)$ \\
\hline \multicolumn{2}{|l|}{ Monito rizacão in suficiente do tratamento } \\
\hline - Falta de controles analíticos & $3(8)$ \\
\hline Medicamento deteriorado & $1(2)$ \\
\hline Falta de adesäo do paciente & $1(2)$ \\
\hline Total & $42(100$ \\
\hline
\end{tabular}

Fonte: Dados da Gerência de Risco do Centro de Transplante de Medula Óssea, 2008-2018.

Na categoria causas da ocorrência do EM, o fator humano foi o mais frequente, $69 \%$ das ocorrências, sendo categorizado pelo conhecimento deficiente, desempenho deficiente, erro no cálculo da dose e infusão, falha na utilização de tecnologia para prescrição dos medicamentos, erro na estocagem, erro no preparo do medicamento, erro de transcrição, estresse, fadiga e comportamento intimidador. A comunicação foi responsável por $21 \%$ dos casos e está relacionada à falha na comunicação verbal e escrita. E 10\% dos casos foram devidos a fatores relacionados ao sistema, que se configura como: iluminação, ruído, interrupções, dimensionamento, pessoal inexperiente, políticas e procedimentos (Tab.III). 
Tabela III - Causas da ocorrência de erros de medicação, Rio de Janeiro, 2018.

\begin{tabular}{ll}
\hline Causas da ocorrência de erros de medicação & \\
\hline Fator humano & $\mathrm{N}$ \\
\hline - Conhecimento deficiente & 4 \\
- Desempenho deficiente & 10 \\
- Erro no cálculo da dose e infusão & 1 \\
- Falha na utilização de tecnologia para prescrição & 5 \\
$\quad$ dos medicamentos & \\
- Erro no preparo do medicamento & \\
\hline Comunicação & 3 \\
\hline - Falha na comunicação verbal & 6 \\
\hline Falha na comunicação escrita & \\
\hline Fatores relacionados ao processo & 4 \\
\hline - Procedimentos
\end{tabular}

Fonte: Dados da Gerência de Risco do Centro de Transplante de Medula Óssea, 2008-2018.

Quanto as consequências para os pacientes, foram identificados eventos adversos: leves $34(82 \%)$; moderados $6(14 \%)$; graves $1(2 \%)$ e fatais $1(2 \%)$. Algumas das consequências descritas foram: modificações do regime de condicionamento; alteração do protocolo de tratamento; agitação psicomotora, falha na mobilização das células-tronco hematopoéticas e óbito.

Em relação ao processo de avaliação dos EM para implementação de melhorias, a figura 1 apresenta uma descrição do fluxo na unidade de Transplante de Medula Óssea.
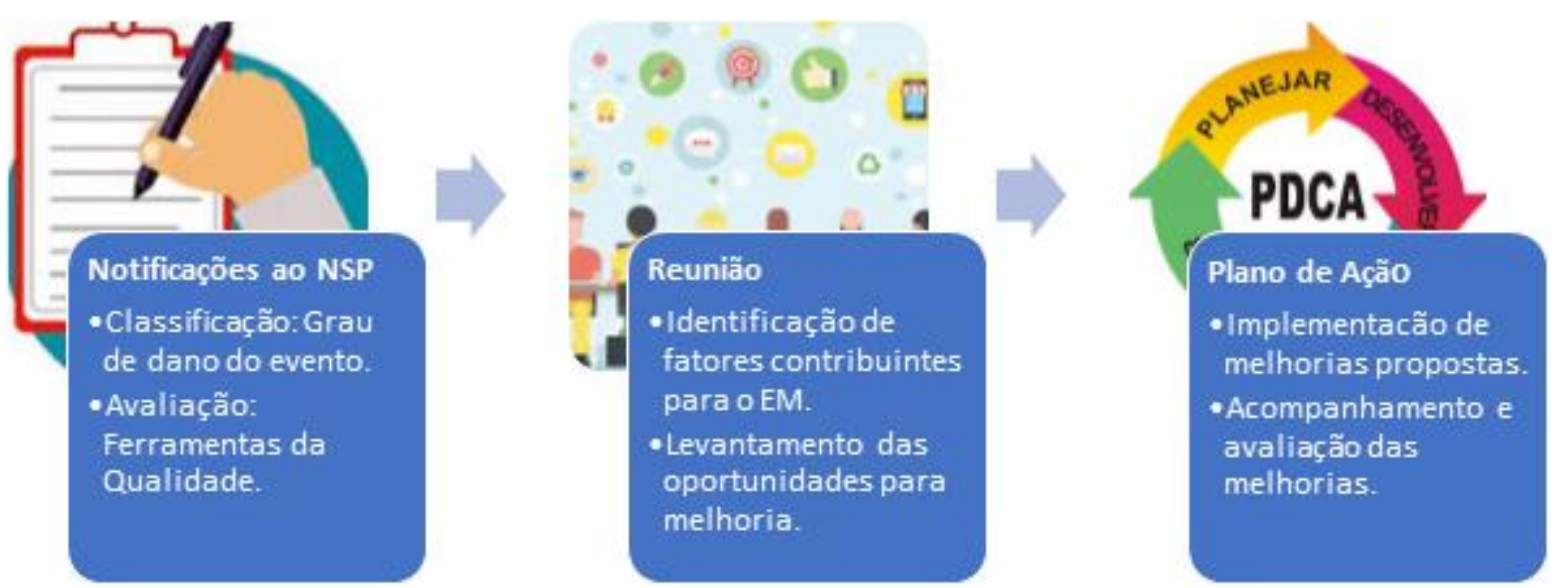

Figura 1 - Fluxo das notificações para o Núcleo de Segurança do Paciente (NSP).

Diante dos EM ocorridos, as ações de melhoria implementadas pela Unidade para prevenção foram: revisão do processo de trabalho desde a dispensação de medicamentos da farmácia até a administração no paciente; treinamento da equipe multidisciplinar; elaboração de procedimento operacional padrão (POP) para dispensação de quimioterápicos; orientação de pacientes e acompanhantes quanto a terapia medicamentosa; revisão do sistema eletrônico de prescrição; treinamento da equipe de enfermagem para dupla checagem da prescrição; orientação para aprazamento por enfermeiras com tempo de experiência no preparo e na administração de quimioterápicos; diminuição do ruído no posto de enfermagem; alerta de alergia na prescrição eletrônica e atualização e treinamento em boas práticas na administração de medicamento.

\section{Discussão}

O transplante de medula óssea abrange a prescrição simultânea de quimioterapia em altas doses e medicamentos de suporte com margem terapêutica reduzida. O que em geral, pode propiciar o erro de medicação [9]. Desta forma, o gerenciamento de medicamentos nesta modalidade terapêutica torna-se um ponto crucial e requer intervenções de enfermagem, por meio de estratégias que garantam uma terapêutica medicamentosa segura. 
Nesta perspectiva, avaliar os EM na unidade de Transplante de Medula Óssea, identificando as consequências para os pacientes e apresentar as ações de melhoria empregadas vai ao encontro do terceiro desafio lançado pela Organização Mundial de Saúde (OMS) em 2017, Global Patient Safety Challenge on Medication Safety, que visa reduzir em $50 \%$ os danos graves e evitáveis associados a medicamentos em todos os países nos próximos cinco anos [3].

Sendo assim, buscando ampliar o conhecimento nesta área, esse estudo apresenta uma série de 42 casos, representando a maior série de casos descrita na literatura científica até o momento. Uma revisão sistemática sobre o assunto, mostrou que as informações sobre os EM em TMO ainda são ínfimas, foram identificados 11 estudos, sendo oito relatos de caso com um quantitativo máximo de três pacientes, dificultando com isso a extrapolação e generalização dos resultados [10].

No presente estudo, o quantitativo da população adulta e pediátrica foram quase que equivalentes, com um percentual de diferença de $8 \%$. Porém, quando se trata de pacientes pediátricos a incidência de EM é três vezes maior. Uma das causas pode ser o fato de $80 \%$ dos fármacos comercializados serem destinados a adultos, dificultando o uso em pediatria devido à necessidade de administração de doses fracionadas com uma manipulação excessiva das soluções e expondo esta população a um risco elevado para ocorrência de erros de medicação $[11,12]$. Outra população que também tem um alto risco para ocorrência de EM são os idosos. Eles representam um grupo-alvo prioritário para implementação de estratégias de prevenção de erros de medicação, devido à presença de comorbidades que tendem a potencializar alterações fisiológicas e consequentes mudanças no perfil farmacocinético e farmacodinâmico de inúmeros fármacos [13-15].

Logo, a educação de pacientes, familiares e cuidadores é fundamental para alcançar dois dos principais objetivos do $3^{\circ}$ Desafio Global de Segurança do Paciente: o empoderamento de pacientes, familiares e cuidadores a participar ativamente e de forma engajada nas decisões relacionadas à assistência à saúde. E o engajamento dos setores envolvidos, parceiros e indústria para sensibilizá-los quanto aos problemas de segurança na medicação $[16,17]$.

A via de administração também foi a mais envolvida na ocorrência EM, neste estudo 59\% dos EM ocorreram pela via endovenosa. Os medicamentos administrados por essa via requerem maior necessidade de ajustes e monitorização ao longo do período. Estima-se que $90 \%$ dos pacientes hospitalizados recebam medicamentos pela via intravenosa por meio de bombas de infusão [18]. No TMO a utilização da via intravenosa e de cateteres centrais para a terapia farmacológica em pacientes submetidos ao transplante é essencial, devido à quantidade de medicamentos administrados e a natureza hostil dos mesmos [18-20].

Esse estudo também identificou os profissionais que estiveram envolvidos nos EM. Os enfermeiros foram citados em $38 \%$ dos casos e os farmacêuticos em $36 \%$, corroborando outro estudo que destacou que o determinante do erro está presente em mais de um subsistema. Em sua análise do conteúdo, os erros classificados como de omissão e de horário estavam relacionados não apenas aos fatores intrínsecos à equipe de enfermagem, mas, também, a fatores associados à distribuição de medicamentos pela farmácia e aos prescritores (preenchimento inadequado de formulários de controle especial para antimicrobianos). Esses resultados refletem a necessidade de otimizar os sistemas de medicação, revisando os subsistemas, reduzindo etapas e simplificando os processos, visando a diminuição destes erros [21].

Em relação aos medicamentos envolvidos no EM, neste estudo, os agentes antineoplásicos e imunossupressores foram os mais frequentes, uma revisão sistemática também identificou o bussulfano (antineoplásico) e a ciclosporina (imunossupressor) na maioria dos erros de medicação no TMO [10]. Estes medicamentos são de índice terapêutico reduzido e foram relacionados na lista de medicamentos de alta vigilância, publicada pela Institute for Safe Medication Practices (ISMP), assim exigem protocolos especiais, como a padronização de pedidos, armazenamento, preparação e administração, entre outros para reduzir o risco de erros $[22,23]$.

Quanto aos tipos e subtipos de EM, neste estudo, os mais frequentes foram: omissão de dose ou do medicamento, dose errada e erro de preparo, manipulação e/ou acondicionamento. A omissão de dose e dose errada são os mais relatados em estudos sobre esta temática [14,2427]. Um estudo realizado no Quebec em uma unidade de terapia intensiva avaliou os incidentes ocorridos na unidade e verificou que $44,3 \%$ eram relacionados a medicação, sendo os erros de omissão na administração os mais comuns, representando $31,1 \%$ do total [28]. Outro estudo analisou 961 prescrições de 90 pacientes e identificou 650 erros de medicação (uma média de 7 
erros por paciente), sendo o erro de omissão do medicamento prescrito o mais frequente. A longa permanência dos profissionais de enfermagem em seus postos de trabalho foi identificada neste estudo como um fator de risco associado ao erro de omissão [14].

As causas da ocorrência de EM são multifatorias [10,29], no presente estudo o fator humano foi o mais frequente, sendo a subcategoria desempenho deficiente a mais elevada. Entende-se como desempenho deficiente a forma ou as condições como são realizadas as atividades relacionadas à terapêutica medicamentosa e não necessariamente ao conhecimento inerente à sua realização [30]. Muitos aspectos interferem no desempenho dos profissionais e há evidências de que a carga de trabalho, como já mencionado e a taxa diária de ocupação são fatores relevantes na gênese dos EM [31].

Os incidentes com medicação estão entre os eventos adversos que mais comumente repercutem na vida do paciente, de seus familiares, dos profissionais de saúde e da sociedade. Nos Estados Unidos, os EM causam pelo menos uma morte todos os dias e prejudicam aproximadamente 1,3 milhão de pessoas anualmente. Estima-se que o erro assistencial seja a terceira causa de morte nos EUA, estando atrás somente de doenças cardiovasculares e câncer, podendo chegar a 400.000 óbitos por ano [17].

As implicações do EM para o paciente podem ser as mais diversas possíveis. Nesse estudo com 42 casos de EM, foram identificadas consequências leves, moderadas, graves e fatais para os pacientes. O ISMP alerta para o cálculo da dose dos antineoplásicos, pois precisa ser individualizado, considerando parâmetros laboratoriais e clínicos, como superfície corporal e tolerância às reações adversas [22,23]. As doses baixas podem ocasionar falha terapêutica, este fato foi observado em um dos nossos relatos com a falha na mobilização das células-tronco hematopoéticas. E a sobredose pode resultar em efeitos tóxicos. Entre as consequências mais graves relacionadas à toxicidade estão a neutropenia grave, trombocitopenia, estomatite grave, mucosite, nefrotoxicidade, hepatotoxicidade, cardiotoxicidade e neurotoxicidade [22,23]. Efeitos neurotóxicos foram relatados em nossa série de casos, como a agitação psicomotora. Entretanto, nem todos os efeitos adversos foram identificados, para que fosse possível fazer uma discussão mais abrangente.

Estudos apontam que os EM são um problema de saúde pública e afetam a qualidade da assistência prestada. Logo, avaliar estes eventos e buscar evidências científicas para prevenilos pode ser o caminho para não incorrer no erro novamente. Uma estratégia é envolver a equipe na notificação dos eventos e discutir fatores de riscos para que ações preventivas sejam implantadas [10,18,20,24,30,32].

As ações preventivas podem ampliar a segurança na administração de medicamentos, logo, destaca-se a necessidade de estratégias como a padronização de processos, o uso de recursos de tecnologia da informação, educação permanente e, principalmente, o monitoramento das práticas profissionais em todas as etapas do processo que envolve o medicamento, visando à prevenção desses eventos [3,10,21].

Também configuram-se como "medidas de prevenção" a valorização de estratégias que fortaleçam as barreiras ao erro de medicação, como a dupla checagem $[18,33]$. Esta, certamente é uma recomendação bastante utilizada, porém faz-se necessário o reconhecimento das limitações quando aplicada como estratégia isolada na prevenção de erros de medicação. Contudo, sua aplicabilidade é bem aceita em controles específicos, como, na programação de bombas infusoras, conferência de dados em pacientes pediátricos e idosos, processo de dispensação e administração de antineoplásicos e na administração em cuidados da terapia intensiva [34].

A estratégia de confirmação dos cinco certos da administração configura-se como uma medida de prevenção. Sendo checados os seguintes itens: paciente certo, medicação certa, dose certa, via certa e o tempo certo, cada um dos "certos" deve funcionar como uma efetiva barreira para evitar as ocorrências de eventos indesejados ao paciente [35]. Esta verificação não deve se restringir somente a enfermagem. Outros autores apresentam os dez certos, enfatizando a responsabilidade da equipe, pacientes e cuidadores. Os dez certos correspondem a paciente certo, medicação certa, dose certa, via certa, horário certo, direito a recusa, direito de esclarecimento, direito de questionamento, orientação correta e direito a informação sobre a eficácia e efeitos do tratamento $[36,37]$.

Logo, o engajamento de profissionais de saúde, gestores, indústria farmacêutica no processo de terapia medicamentosa e o empoderamento de pacientes, familiares e cuidadores para que participem ativamente nas decisões relacionadas aos seus cuidados em saúde. Esta participação dar-se-á por meio de perguntas, identificando erros e gerenciando seus 
medicamentos, são ações que podem representar a última barreira de prevenção de um erro no cuidado em saúde [25].

Em relação as limitações, por se tratar de um estudo retrospectivo, não foi possível entrevistar cada membro envolvido no EM para avaliar com mais precisão as causas e as consequências do mesmo. Os possíveis eventos adversos não foram descritos em todas as notificações, o que dificultou a análise desse dado.

\section{Conclusão}

Emergiu deste estudo a realidade complexa da terapia medicamentosa na unidade de transplante de medula óssea, foram identificados os erros de medicação que põem em risco a segurança do paciente. Os tipos de EM identificados foram semelhantes com os de outras unidades, principalmente aos da terapia intensiva. Os medicamentos envolvidos nos EM com maior possibilidade de danos ao paciente foram os agentes antineoplásicos. Logo, estratégias para prevenir os erros com esses medicamentos foram descritas e cabem para todas as unidades de tratamento que cuidem de pacientes oncológicos.

As causas dos EM são multifatoriais, devendo ser enfrentadas com uma visão holística da situação, utilizando estratégias múltiplas como os dez certos. As consequências destes erros podem ser graves e fatais para pacientes, o que exige dos profissionais de saúde ações mais efetivas que tenham uma acurácia maior para prevenir os EM. Logo, a administração de medicamentos não é uma tarefa simples e exige julgamento clínico dos profissionais envolvidos no processo, assim como o acompanhamento do paciente durante todo tratamento.

O conhecimento sobre farmacocinética, farmacodinâmica, reações adversas, interações medicamentosas e conciliação medicamentosa pode contribuir para segurança do paciente, visto que nenhum profissional de saúde deveria administrar um medicamento sem conhecer seus efeitos, sem estarem seguros para fazê-lo ou quando não forem capazes de explicá-lo ao paciente. Sendo assim, os mecanismos de prevenção dos erros têm que obrigatoriamente passar por todos os profissionais envolvidos no sistema de medicação, para que todos sejam igualmente responsáveis pelo desfecho. A assistência de saúde é, majoritariamente, um trabalho coletivo no qual as responsabilidades também devem ser compartilhadas, inclusive com pacientes e seus cuidadores.

\section{Conflitos de interesse}

Os autores declaram ausência de conflitos de interesses.

\section{Financiamento}

O trabalho foi realizado com recursos próprios dos investigadores.

1. Magalhães AMM, Moura GMSS, Pasin SS, Funcke LB, Pardal BM, Kreling A.

Processos de medicação, carga de trabalho e a segurança do paciente em unidades de internação. Rev Esc Enferm USP 2015;49:43-50. https://doi.org/10.1590/S0080623420150000700007

2. Jha AK, World Health Organization, World Alliance for Patient Safety, Research Priority Setting Working Group. Summary of the evidence on patient safety: implications for research. Geneva, Switzerland: World Health Organization; 2008.

3. World Health Organization. Medication without harm - Global Patient Safety Challenge on Medication Safety; 2017.

4. Brasil MS. Documento de referência para o Programa Nacional de Segurança do Paciente 2014.

5. ANVISA. Gestão de riscos e investigação de eventos adversos relacionados à assistência à saúde; Brasília: Anvisa; 2017.

6. Fonseca RB, Secoli SR. Medicamentos utilizados em transplante de medula óssea: um estudo sobre combinações dos antimicrobianos potencialmente interativos. Rev Esc Enferm USP 2008;42:706-14. https://doi.org/10.1590/S0080-62342008000400013 
7. Otero López MJ, Castaño Rodríguez B, Pérez Encinas M, Codina Jané C, Tamés Alonso MJ, Sánchez Muñoz T. Actualización de la clasificación de errores de medicación del grupo Ruiz-Jarabo 2000. Farmacia Hospitalaria 2008;32:38-52. https://doi.org/10.1016/S1130-6343(08)72808-3

8. Ministério da Saúde. Resolução 466/12: Trata de pesquisas em seres humanos e atualiza a resolução 196. Brasília: MS; 2012.

9. Guastaldi R, Secoli S. Interações medicamentosas de antimicrobianos utilizados em transplante de células-tronco hematopoéticas. Rev Latinoam Enferm 2011;19(4):960-7. https://doi.org/10.1590/S0104-11692011000400015

10. Lermontov SP, Carreiro BS, Rezende CM. Medication errors in the context of hematopoietic stem cell transplantation: a systematic review. Cancer Nursing 2019;42:365-72. https://doi.org/10.1097/NCC.0000000000000613

11. Impicciatore $P$, Choonara I, Clarkson A, Provasi D, Pandolfini C, Bonati M. Incidence of adverse drug reactions in paediatric in/out-patients: a systematic review and metaanalysis of prospective studies: Incidence of adverse drug reactions in children. $\mathrm{Br} \mathrm{J}$ Clin Pharmacol 2001;52:77-83. https://doi.org/10.1046/i.0306-5251.2001.01407.x

12. Belela ASC, Pedreira MLG, Peterlini MAS. Erros de medicação em pediatria. Rev Bras Enferm 2011;64:563-9. https://doi.org/10.1590/S0034-71672011000300022

13. Silva $\mathrm{CH}$, Spinillo CG. Dificuldades e estratégias no uso de múltiplos medicamentos por idosos no contexto do design da informação. Estudos em Design 2016;24:130-44.

14. Suclupe S, Martinez-Zapata MJ, Mancebo J, Font-Vaquer A, Castillo-Masa AM, Viñolas I, et al. Medication errors in prescription and administration in critically ill patients. J Adv Nurs 2020;76:1192-200. https://doi.org/10.1111/jan.14322

15. Boletim ISMP. Medicamentos potencialmente inadequados para idosos. Boletim ISMP; 2017. http://biblioteca.cofen.gov.br/wp-content/uploads/2017/11/Medicamentospotencialmente-inadequados-para-idosos.pdf

16. Boletim ISMP. Desafio global de segurança do paciente medicação sem danos. Boletim ISMP; 2018. http://biblioteca.cofen.gov.br/desafio-global-seguranca-pacientemedicacao-sem-danos/

17. Makary MA, Daniel M. Medical error-the third leading cause of death in the US. BMJ 2016:i2139. https://doi.org/10.1136/bmi.i2139

18. Giuliano KK. Intravenous smart pumps. Critical care nursing clinics of North America 2018;30:215-24. https://doi.org/10.1016/i.cnc.2018.02.004

19. Goldspiel B, Hoffman JM, Griffith NL, Goodin S, De Christoforo R, Montello CM, et al. ASHP Guidelines on Preventing Medication Errors with Chemotherapy and Biotherapy. American Journal of Health-System Pharmacy 2015;72:e6-35. https://doi.org/10.2146/sp150001

20. Bohomol E, Ramos LH, D'Innocenzo M. Medication errors in an intensive care unit. J Adv Nurs 2009;65:1259-67. https://doi.org/10.1111/j.1365-2648.2009.04979.x

21. Silva AEBC, Reis AMM, Miasso Al, Santos JO, Cassiani SHDB. Adverse drug events in a sentinel hospital in the State of Goiás, Brazil. Rev Latinoam Enferm 2011;19:378-86. https://doi.org/10.1590/S0104-11692011000200021

22. Boletim ISMP. Antineoplásicos parenterais: erros de medicação, riscos e práticas seguras na utilização. Boletim ISMP; 2014. https://proqualis.net/boletim/antineopl\%C3\%A1 sicos-parenterais-erros-demedica\%C3\%A7\%C3\%A3o-riscos-e-pr\%C3\%A1ticas-seguras-nautiliza\%C3\%A7\%C3\%A30

23. Institute for Safe Medication Practices. ISMP list of high-alert medications in acute care settings. 2018. https://www.ismp.org/recommendations/high-alert-medications-acute-list

24. Néri EDR, Gadêlha PGC, Maia SG, Pereira AGS, Almeida PC, Rodrigues CRM, et al. Drug prescription errors in a Brazilian hospital. Rev Assoc Med Bras (1992) 2011;57:301-8. https://doi.org/10.1016/S2255-4823(11)70063-7

25. Santos PRA, Rocha FLR, Sampaio CSJC. Ações para segurança na prescrição, uso e administração de medicamentos em unidades de pronto atendimento. Rev Gaúcha Enferm 2019;40:e20180347. https://doi.org/10.1590/1983-1447.2019.20180347

26. Heneka N, Shaw T, Rowett D, Lapkin S, Phillips JL. Exploring factors contributing to medication errors with opioids in Australian specialist palliative care inpatient services: A multi-incident analysis. J Palliat Med 2018;21:825-35. https://doi.org/10.1089/jpm.2017.0578 
27. Keers RN, Williams SD, Cooke J, Ashcroft DM. Prevalence and nature of medication administration errors in health care settings: A systematic review of direct observational evidence. Ann Pharmacother 2013;47:237-56. https://doi.org/10.1345/aph.1R147

28. Poder TG, Maltais S. Systemic analysis of medication administration omission errors in a tertiary-care hospital in Quebec. Health Information Management Journal 2018:183335831878109. https://doi.org/10.1177/1833358318781099

29. Baraki Z, Abay M, Tsegay L, Gerensea H, Kebede A, Teklay H. Medication administration error and contributing factors among pediatric inpatient in public hospitals of Tigray, northern Ethiopia. BMC Pediatr 2018;18:321. https://doi.org/10.1186/s12887018-1294-5

30. Yamamoto MS, Peterlini MAS, Bohomol E. Notificação espontânea de erros de medicação em hospital universitário pediátrico. Acta Paul Enferm 2011;24:766-71. https://doi.org/10.1590/S0103-21002011000600006

31. Lerner RBME, Carvalho M, Vieira AA, Lopes JMA, Moreira MEL. Medication errors in a neonatal intensive care unit. J Pediatr (Rio J) 2008;84:166-70. https://doi.org/10.2223/JPED.1757

32. Rosa MB, Perini E, Anacleto TA, Neiva HM, Bogutchi T. Erros na prescrição hospitalar de medicamentos potencialmente perigosos. Rev Saúde Pública 2009;43:490-8. https://doi.org/10.1590/S0034-89102009005000028

33. Teixeira TCA, Cassiani SHDB. Análise de causa raiz: avaliação de erros de medicação em um hospital universitário. Rev Esc Enferm USP 2010;44:139-46. https://doi.org/10.1590/S0080-62342010000100020

34. Neuss MN, Gilmore TR, Belderson KM, Billett AL, Conti-Kalchik T, Harvey BE et al. 2016 Updated American Society of Clinical Oncology/Oncology Nursing Society Chemotherapy Administration Safety Standards, Including Standards for Pediatric Oncology. JOP 2016;12:1262-71. https://doi.org/10.1200/JOP.2016.017905

35. Elliott M, Liu Y. The nine rights of medication administration: an overview. Br J Nurs 2010;19:300-5. https://doi.org/10.12968/bjon.2010.19.5.47064

36. Edwards S, Axe S. The 10 'R's of safe multidisciplinary drug administration. Nurse Prescribing 2015;13:398-406. https://doi.org/10.12968/npre.2015.13.8.398

37. Kavanagh $\mathrm{C}$. Medication governance: preventing errors and promoting patient safety. Br J Nurs 2017;26:159-65. https://doi.org/10.12968/bjon.2017.26.3.159 